Kragujevac Journal of Mathematics

Volume 41(1) (2017), Pages 71-80.

\title{
CARLEMAN INTEGRAL OPERATORS AS MULTIPLICATION OPERATORS AND PERTURBATION THEORY
}

\author{
S. M. BAHRI ${ }^{1}$
}

\begin{abstract}
In this paper we introduce a multiplication operation that allows us to give to the Carleman integral operator of second class $[3,8]$ the form of a multiplication operator. Also we establish the formaly theory of perturbation of such operators.
\end{abstract}

\section{INTRODUCTION}

It is well known that the multiplication operators $[1,2]$ possess a very rich structure theory, such that these operators played an important role in the study of operators on Hilbert Spaces.

In this paper, we introduce a multiplication operation that allows us to give to the Carleman integral operator of second class $[3,8]$ the form of a multiplication operator.

In what follows, we shall assume that the reader is familiar with the fundamental results and the standard notation of the Integral operators theory [8-12]. Let $X$ be an arbitrary set, $\mu$ a $\sigma$-finite measure on $X$ ( $\mu$ is defined on a $\sigma$-algebra of subsets of $X$, we don't indicate this $\sigma$-algebra), $L_{2}(X, \mu)$ the Hilbert space of square integrable functions with respect to $\mu$. Instead of writing " $\mu$-measurable", " $\mu$-almost everywhere" and " $(d \mu(x))$ " we write "measurable", "a.e." and " $d x$ ".

A linear operator $A: D(A) \longrightarrow L_{2}(X, \mu)$, where the domain $D(A)$ is a dense linear manifold in $L_{2}(X, \mu)$, is said to be integral if there exists a measurable function $K$ on $X \times X$, a kernel, such that, for every $f \in D(A)$,

$$
A f(x)=\int_{X} K(x, y) f(y) d y \text { a.e. }
$$

Key words and phrases. Carleman kernel, defect indices, integral operator, formal series. 2010 Mathematics Subject Classification. Primary: 45P05, 47B25. Secondary: 13F25.

Received: May 7, 2016.

Accepted: August 10, 2016. 
A kernel $K$ on $X \times X$ is said to be Carleman if $K(x, y) \in L_{2}(X, \mu)$ for almost every fixed $x$, that is to say

$$
\int_{X}|K(x, y)|^{2} d y<\infty \text { a.e. }
$$

An integral operator $A$ with a kernel $K$ is called Carleman operator if $K$ is a Carleman kernel. Every Carleman kernel $K$ defines a Carleman function $k$ from $X$ to $L_{2}(X, \mu)$ by $k(x)=\overline{K(x, \cdot)}$ for all $x$ in $X$ for which $K(x, \cdot) \in L_{2}(X, \mu)$.

Now we consider the Carleman integral operator (1.1) of second class [3,8] generated by the following symmetric kernel

$$
K(x, y)=\sum_{n=0}^{\infty} a_{n} \psi_{n}(x) \overline{\psi_{n}(y)}
$$

where the overbar denotes the complex conjugation, $\left(\psi_{n}(x)\right)_{n=0}^{\infty}$ is an orthonormal sequence in $L^{2}(X, \mu)$ such that

$$
\sum_{n=0}^{\infty}\left|\psi_{n}(x)\right|^{2}<\infty \text { a.e. }
$$

and $\left(a_{n}\right)_{n=0}^{\infty}$ is a real number sequence verifying

$$
\sum_{n=0}^{\infty} a_{n}^{2}\left|\psi_{n}(x)\right|^{2}<\infty \text { a.e. }
$$

We call $\left(\psi_{n}(x)\right)_{n=0}^{\infty}$ a Carleman sequence.

Moreover, we assume that there exists a numeric sequence $\left(\gamma_{n}\right)_{n=0}^{\infty}$ such that

$$
\sum_{n=0}^{\infty} \gamma_{n} \psi_{n}(x)=0 \text { a.e., }
$$

and

$$
\sum_{n=0}^{\infty}\left|\frac{\gamma_{n}}{a_{n}-\lambda}\right|^{2}<\infty
$$

With the conditions (1.2) and (1.3), the symmetric operator $A=\left(A^{*}\right)^{*}$ admits the defect indices $(1,1)$ (see [3-6]), and its adjoint operator is given by

$$
\begin{aligned}
A^{*} f(x) & =\sum_{n=0}^{\infty} a_{n}\left(f, \psi_{n}\right) \psi_{n}(x), \\
D\left(A^{*}\right) & =\left\{f \in L^{2}(X, \mu): \sum_{n=0}^{\infty} a_{n}\left(f, \psi_{n}\right) \psi_{n}(x) \in L^{2}(X, \mu)\right\} .
\end{aligned}
$$

Moreover, we have

$$
\left\{\begin{array}{l}
\varphi_{\lambda}(x)=\sum_{n=0}^{\infty} \frac{\gamma_{n}}{a_{n}-\lambda} \psi_{n}(x) \in \mathfrak{N}_{\bar{\lambda}}, \quad \lambda \in \mathbb{C}, \lambda \neq a_{n}, n=1,2, \ldots, \\
\varphi_{a_{n}}(x)=\psi_{n}(x)
\end{array}\right.
$$


$\mathfrak{N}_{\bar{\lambda}}$ being the defect space associated to $\lambda$ (see $[3,4]$ ).

\section{Position Operator}

Let $\psi=\left(\psi_{n}\right)_{n=0}^{\infty}$ be a fixed Carleman sequence in $L^{2}(X, \mu)$. It is clear from the foregoing that $\psi$ is not a complete sequence in $L^{2}(X, \mu)$. We set $\mathfrak{L}_{\psi}$ the closure of the linear span of the sequence $\left(\psi_{n}(x)\right)_{n=0}^{\infty}$

$$
\mathfrak{L}_{\psi}=\overline{\operatorname{span}\left\{\psi_{n}: n \in \mathbb{N}\right\}}
$$

We start this section by defining some formaly spaces.

\subsection{Formal Elements.}

Definition 2.1 ([7]). We call formal element any expression of the form

$$
f=\sum_{n \in \mathbb{N}} a_{n} \psi_{n}
$$

where the coefficients $a_{n}(n \in \mathbb{N})$ are scalars.

The sequence $\left(a_{n}\right)_{n}$ is said to generate the formal element $f$.

Definition 2.2. We say that $f$ is the zero formal element and we note $f=0$ if $a_{n}=0$ for all $n \in \mathbb{N}$.

We say that two formal elements $f=\sum_{n \in \mathbb{N}} a_{n} \psi_{n}$ and $g=\sum_{n \in \mathbb{N}} b_{n} \psi_{n}$ are equal if $a_{n}=b_{n}$ for all $n \in \mathbb{N}$.

If $\varphi$ is a scalar function defined for each $a_{n}$, we set

$$
\varphi\left(\sum_{n} a_{n} \psi_{n}\right)=\sum_{n} \varphi\left(a_{n}\right) \psi_{n}
$$

or in another form,

$$
\varphi\left(a_{1}, a_{2}, \ldots, a_{n}, \ldots\right)=\left(\varphi\left(a_{1}\right), \varphi\left(a_{2}\right), \ldots, \varphi\left(a_{n}\right), \ldots\right) .
$$

For example let

$$
\varphi(x)=\frac{1}{x}, \quad x \neq 0 .
$$

If $a_{n} \neq 0$ for all $n \in \mathbb{N}$, then the formal element

$$
\varphi\left(\sum_{n} a_{n} \psi_{n}\right)=\sum_{n} \frac{1}{a_{n}} \psi_{n}
$$

is called inverse of the formal element $f=\sum_{n} a_{n} \psi_{n}$.

Furthermore, we define the conjugate of a formal element $f$ by

$$
\bar{f}=\sum_{n} \overline{a_{n}} \psi_{n}
$$

Denotes by $\mathcal{F}_{\psi}$ the set of all formal elements (2.1). On $\mathcal{F}_{\psi}$, we define the following algebraic operations: 
(a) the sum

$$
+: \begin{array}{ccc}
\mathcal{F}_{\psi} \times \mathcal{F}_{\psi} & \rightarrow & \mathcal{F}_{\psi} \\
\left(\sum_{n} a_{n} \psi_{n}\right)+\left(\sum_{n} b_{n} \psi_{n}\right) & = & \sum_{n}\left(a_{n}+b_{n}\right) \psi_{n}
\end{array},
$$

(b) and the product

$$
\begin{array}{ccc}
\mathbb{C} \times \mathcal{F}_{\psi} & \rightarrow & \mathcal{F}_{\psi} \\
\lambda \cdot\left(\sum_{n} a_{n} \psi_{n}\right) & = & \sum_{n}\left(\lambda \cdot a_{n}\right) \psi_{n} .
\end{array}
$$

Hence, we obtain a complex vector space structure for $\mathcal{F}_{\psi}$.

\subsection{Bounded Formal Elements.}

Definition 2.3. A formal element $f=\sum_{n \in \mathbb{N}} a_{n} \psi_{n}$ is bounded if its sequence $\left(a_{n}\right)_{n}$ is bounded.

We denote by $\mathcal{B}_{\psi}$ the set of all bounded formal elements. It's clear that $\mathcal{B}_{\psi}$ is a subspace of $\mathcal{F}_{\psi}$. We claim that:

(a) $\mathcal{L}_{\psi}$ is a subspace of $\mathcal{B}_{\psi}$.

(b) Furthermore we have the strict inclusions:

$$
\mathcal{L}_{\psi} \subset \mathcal{B}_{\psi} \subset \mathcal{F}_{\psi}
$$

We define a linear form $\langle\cdot, \cdot\rangle$ on $\mathcal{F}_{\psi}$ by setting

$$
\left\langle\sum_{n} a_{n} \psi_{n}, \sum_{n} b_{n} \psi_{n}\right\rangle=\sum_{n} a_{n} \overline{b_{n}},
$$

with the series converging on the right side.

Proposition 2.1. The form (2.2) verifies the properties of scalar product.

Proof. Indeed, let

$$
f=\sum_{n} a_{n} \psi_{n}, \quad g=\sum_{n} b_{n} \psi_{n}, \quad f_{1}=\sum_{n} a_{n}^{1} \psi_{n} \quad \text { and } f_{2}=\sum_{n} a_{n}^{2} \psi_{n},
$$

in $\mathcal{F}_{\psi}$.

We have then:

(a)

(b)

$$
\langle f, g\rangle=\sum_{n} a_{n} \overline{b_{n}}=\overline{\sum_{n} b_{n} \overline{a_{n}}}=\overline{\langle g, f\rangle}
$$

$$
\begin{aligned}
\langle\lambda f, g\rangle & =\left\langle\lambda\left(\sum_{n} a_{n} \psi_{n}\right), \sum_{n} a_{n} \psi_{n}\right\rangle=\left\langle\sum_{n}\left(\lambda a_{n}\right) \psi_{n}, \sum_{n} b_{n} \psi_{n}\right\rangle \\
& =\sum_{n}\left(\lambda a_{n}\right) \overline{b_{n}}=\lambda\left\langle\sum_{n} a_{n} \psi_{n}, \sum_{n} b_{n} \psi_{n}\right\rangle=\lambda\langle f, g\rangle,
\end{aligned}
$$


(c)

$$
\begin{aligned}
\left\langle f_{1}+f_{2}, g\right\rangle & =\left\langle\sum_{n}\left(a_{n}^{1}+a_{n}^{2}\right) \psi_{n}, \sum_{n} b_{n} \psi_{n}\right\rangle \\
& =\sum_{n}\left(a_{n}^{1}+a_{n}^{2}\right) \overline{b_{n}}=\sum_{n} a_{n}^{1} \overline{b_{n}}+\sum_{n} a_{n}^{2} \overline{b_{n}}=\left\langle f_{1}, g\right\rangle+\left\langle f_{2}, g\right\rangle,
\end{aligned}
$$

(d)

$$
\langle f, f\rangle=\sum_{n}\left|a_{n}\right|^{2} \geq 0 \text { and }\langle f, f\rangle>0, \quad \text { if } f \neq 0 .
$$

Remark 2.1. On $\mathcal{L}_{\psi}$, the scalar product $\langle.,$.$\rangle coincides with the scalar product (.,$. of $L^{2}(X, \mu)$.

2.3. The Multiplication Operation. Here, we introduce the crucial tool of our work.

Definition 2.4. We call multiplication with respect to the Carleman sequence $\left(\psi_{n}\right)_{n}$, the operation denoted "o" and defined by

$$
f \circ g=\sum_{n}\left\langle f, \psi_{n}\right\rangle\left\langle g, \psi_{n}\right\rangle \psi_{n}=\sum_{n} a_{n} b_{n} \psi_{n}, \quad \text { for all }(f, g) \in \mathcal{F}_{\psi}^{2} .
$$

Definition 2.5. We call position operator in $\mathcal{L}_{\psi}$ any unitary selfadjoint operator satisfying

$$
U(f \circ g)=U f \circ U g, \quad \text { for all } f, g \in \mathcal{L}_{\psi} .
$$

The term "position operator" comes from the fact (as it will be shown in the following theorem) that for the elements of the sequence $\psi=\left(\psi_{n}\right)_{n}$, the operator $U$ acts as operator of change of position of these elements.

\subsection{Main Results.}

Theorem 2.1. A linear operator defined on $\mathcal{L}_{\psi}$ is a position operator if and only if there exists an involution $j$ (i.e. $j^{2}=I d$ ) of the set $\mathbb{N}$ such that for all $n \in \mathbb{N}$

$$
U \psi_{n}=\psi_{j(n)} \text {. }
$$

Proof.

(a) It is easy to see that if (2.3) holds, then $U$ is a position operator.

(b) Let $U$ be a position operator. According to Proposition 2.1 we can write:

$$
U \psi_{n}=\sum_{k} \alpha_{n, k} \psi_{k}, \quad \text { with } \sum_{k}\left|\alpha_{n, k}\right|^{2}=1
$$

since $U \psi_{n} \in \mathcal{L}_{\psi}$.

On the other hand, we have

$$
\sum_{k} \alpha_{n, k} \psi_{k}=\sum_{k} \alpha_{n, k}^{2} \psi_{k}
$$


as

$$
U \psi_{n}=U\left(\psi_{n} \circ \psi_{n}\right)=U \psi_{n} \circ U \psi_{n} .
$$

The equalities (2.4) lead to the resolution of the system:

$$
\left\{\begin{array}{l}
\sum_{n}\left|\alpha_{n, k}\right|^{2}=1, \\
\alpha_{n, k}^{2}=\alpha_{n, k},
\end{array} \quad k \in \mathbb{N} .\right.
$$

We get then

for all $n \in \mathbb{N}$, there exists only one $k_{n} \in \mathbb{N}:\left\{\begin{array}{l}\alpha_{n, k_{n}}=1, \\ \alpha_{n, k}=0,\end{array} \quad\right.$ for all $k \neq k_{n}$.

Let us now consider the following application

$$
\begin{aligned}
j: \mathbb{N} & \rightarrow \mathbb{N}, \\
n & \mapsto j(n)=k_{n} .
\end{aligned}
$$

It's clear that $j$ is injective.

Now let $m \in \mathbb{N}$. Since $U^{2}=I$, then

$$
U\left(U \psi_{m}\right)=U \psi_{j(m)}=\psi_{j(j(m))}=\psi_{m} .
$$

Hence

$$
j(j(m))=m .
$$

Finally $j$ is well defined as involution.

Remark 2.2.

(a) We emphasize that involution $j$ depends of the operator $U$, i.e. $j=j_{U}$. We then write

$$
U \psi_{n}=\psi_{j(n)}=\psi_{j_{U}(n)}
$$

and

$$
U f=U\left(\sum_{n} a_{n} \psi_{n}\right)=\sum_{n} a_{n} \psi_{j(n)}=f_{U}
$$

(b) The position operator $U$ can be extended over $\mathcal{F}_{\psi}$ as follows. If $f=\sum_{n} a_{n} \psi_{n} \in$ $\mathcal{F}_{\psi}$, then

$$
U f=f_{U}=\sum_{n} a_{n} \psi_{j_{U}(n)}
$$

\section{Carleman Operator in $\mathcal{F}_{\psi}$}

3.1. Case of Defect Indices $(\mathbf{1}, \mathbf{1})$. Let $\alpha=\sum_{n} \alpha_{p} \psi_{p} \in \mathcal{F}_{\psi}$, we introduce the operator $\stackrel{\circ}{A}_{\alpha}$ defined in $\mathcal{L}_{\psi}$ by

$$
\stackrel{\circ}{A}_{\alpha} f=\alpha \circ f=\sum_{n}\left\langle\alpha, \psi_{n}\right\rangle\left\langle f, \psi_{n}\right\rangle \psi_{n} .
$$


It is clear that $\stackrel{\circ}{A}_{\alpha}$ is a Carleman operator induced by the kernel

$$
K(x, y)=\sum_{n} \alpha_{n} \psi_{n}(x) \overline{\psi_{n}(y)}
$$

with domain

$$
D\left(\stackrel{\circ}{A}_{\alpha}\right)=\left\{f \in \mathcal{L}_{\psi}: \sum_{n}\left|\alpha_{n}\left(f, \psi_{n}\right)\right|^{2}<\infty\right\}
$$

Moreover, if $\alpha=\bar{\alpha}$, then $\stackrel{\circ}{A}_{\alpha}$ is selfadjoint.

Now let $\Theta=\sum_{n} \gamma_{n} \psi_{n} \in \mathcal{F}_{\psi}$ and $\Theta \notin \mathcal{L}_{\psi}$ (i.e., $\sum_{n}\left|\gamma_{n}\right|^{2}=\infty$ ). We introduce the following set

$$
\mathcal{H}_{\Theta}=\left\{f+\mu \Theta: f \in \mathcal{L}_{\psi}, \mu \in \mathbb{C}\right\},
$$

which verify the following properties.

\section{Proposition 3.1.}

(a) $\mathcal{H}_{\Theta}$ is a subset of $\mathcal{F}_{\psi}$.

(b) $\mathcal{H}_{\theta}=\mathcal{L}_{\psi} \oplus \mathbb{C} \theta$, i.e. direct sum of $\mathcal{L}_{\psi}$ with $\mathbb{C} \theta=\{\mu \theta: \mu \in \mathbb{C}\}$.

Proof. The first property is easy to establish. We show the uniqueness for the second.

Let $g_{1}=f_{1}+\mu_{1} \theta$ and $g_{2}=f_{2}+\mu_{2} \theta$ two formal elements in $\mathcal{H}_{\theta}$. Then

$$
g_{1}=g_{2} \Leftrightarrow f_{1}-f_{2}=\left(\mu_{2}-\mu_{1}\right) \theta
$$

This last equality is verified only if $\mu_{2}=\mu_{1}$. Therefore $f_{1}=f_{2}$.

Denote by $Q$ the projector of $\mathcal{H}_{\Theta}$ on $\mathcal{L}_{\psi}$, that is to say: if $g \in \mathcal{H}_{\Theta}, g=f+\mu \Theta$ with $f \in \mathcal{L}_{\psi}$ and $\mu \in \mathbb{C}$ then $Q g=f$.

We define the operator $B_{\alpha}$ by

$$
B_{\alpha} f=Q(\alpha \circ f), \quad f \in \mathcal{L}_{\psi} .
$$

It is clear that,

$$
D\left(B_{\alpha}\right)=\left\{f \in \mathcal{L}_{\psi}: \alpha \circ f \in \mathcal{H}_{\Theta}\right\}
$$

Theorem 3.1. $B_{\alpha}$ is a densely defined and closed operator. 
Proof.

(a) Since

$$
\operatorname{span}\left\{\psi_{n}: n \in \mathbb{N}\right\} \subset D\left(B_{\alpha}\right)
$$

and that $\left(\psi_{n}\right)_{n}$ is complete in $\mathcal{L}_{\psi}$, then

$$
\overline{D\left(B_{\alpha}\right)}=\mathcal{L}_{\psi} .
$$

(b) Let $\left(f_{n}\right)_{n}$, be a sequence of elements in $D\left(B_{\alpha}\right)$. Checking

$$
\left\{\begin{array}{c}
f_{n} \rightarrow f, \\
B_{\alpha} f_{n} \rightarrow g,
\end{array} \quad \text { (convergence in the } L^{2}\right. \text { sense). }
$$

We have then

$$
B_{\alpha} f_{n}=Q\left(\alpha \circ f_{n}\right)
$$

with

$$
\alpha \circ f_{n}=g_{n}+\mu \Theta, g_{n} \in \mathcal{L}_{\psi}
$$

Then

$$
g_{n}=\alpha \circ f_{n}-\mu_{n} \Theta \in \mathcal{L}_{\psi}
$$

This implies that:

$$
\left\langle g_{n}, \psi_{m}\right\rangle=\alpha_{m}\left\langle f_{n}, \psi_{m}\right\rangle-\mu_{n} \gamma_{m} \psi_{m}, \quad \text { for all } m \in \mathbb{N} .
$$

Or, when $n$ tends to $\infty$, we have:

$$
g_{n} \rightarrow g \text { and } f_{n} \rightarrow f .
$$

Therefore, there exist $\mu \in \mathbb{C}$ such that:

$$
\lim _{n \rightarrow \infty} \mu_{n}=\mu \text {. }
$$

And as $Q$ is a closed operator, then we can write

$$
\alpha \circ f \in \mathcal{H}_{\Theta} \quad \text { and } \quad g=Q(\alpha \circ f) .
$$

Finally $f \in D\left(B_{\alpha}\right)$ and $g=B_{\alpha} f$.

It follows from this theorem that the adjoint operator $B_{\alpha}^{*}$ exists and $B_{\alpha}^{* *}=B_{\alpha}$.

Let us denote by $A_{\alpha}$, the operator adjoint of $B_{\alpha}$

$$
A_{\alpha}=B_{\alpha}^{*}
$$

In the case $\alpha=\bar{\alpha}$, the operator $A_{\alpha}$ is symmetric and we have the following results.

Theorem 3.2. $A_{\alpha}$ admits defect indices $(1,1)$ if and only if

$$
\varphi_{\lambda}=(\alpha-\lambda)^{-1} \circ \Theta \in \mathcal{L}_{\psi} .
$$

In this case $\varphi_{\lambda} \in \mathcal{N}_{\bar{\lambda}}$ (defect space associated with $\lambda$, [3]). 
Proof. We know (see [3]) that $A_{\alpha}$ has the defect indices $(1,1)$ if and only if, its defect subspaces $\mathcal{N}_{\bar{\lambda}}$ and $\mathcal{N}_{\lambda}$ are unidimensional.

We have

$$
\mathcal{N}_{\bar{\lambda}}=\operatorname{ker}\left(A_{\alpha}^{*}-\lambda I\right)=\operatorname{ker}\left(B_{\alpha}-\lambda I\right) .
$$

So it suffices to solve the system

$$
\left\{\begin{array}{l}
B_{\alpha} \varphi_{\lambda}=\lambda \varphi_{\lambda} \\
\varphi_{\lambda} \in \mathcal{L}_{\psi}
\end{array}\right.
$$

i.e.,

$$
\begin{aligned}
\left\{\begin{array}{l}
Q\left(\alpha \circ \varphi_{\lambda}\right)=\lambda \varphi_{\lambda}, \\
\varphi_{\lambda} \in \mathcal{L}_{\psi},
\end{array}\right. & \Longleftrightarrow\left\{\begin{array}{l}
\left(\alpha \circ \varphi_{\lambda}\right)=\lambda \varphi_{\lambda}+\mu \Theta, \mu \in \mathbb{C}, \\
\varphi_{\lambda} \in \mathcal{L}_{\psi},
\end{array}\right. \\
& \Longleftrightarrow\left\{\begin{array}{l}
(\alpha-\lambda) \circ \varphi_{\lambda}=\Theta, \\
\varphi_{\lambda} \in \mathcal{L}_{\psi},
\end{array}\right. \\
& \Longleftrightarrow\left\{\begin{array}{l}
\varphi_{\lambda}=(\alpha-\lambda)^{-1} \circ \Theta, \\
\varphi_{\lambda} \in \mathcal{L}_{\psi} .
\end{array}\right.
\end{aligned}
$$

3.2. Case of Defect Indices $(\mathbf{m}, \mathbf{m})$. In this section we give the generalization for the case of defect indices $(m, m)$, where $m>1$.

Let $\Theta_{1}, \Theta_{2}, \ldots, \Theta_{m},($ where $m \in \mathbb{N})$ formal elements not belonging to $\mathcal{L}_{\psi}$ and let

$$
\mathcal{H}_{\Theta}=\left\{f+\sum_{k=1}^{m} \mu_{k} \Theta_{k}, \quad f \in \mathcal{L}_{\psi}, \mu_{k} \in \mathbb{C}, \quad k=1, \ldots, m\right\} .
$$

We consider the operator $B_{\alpha}$ defined by

$$
\begin{aligned}
B_{\alpha} f & =Q(\alpha \circ f), \quad \text { for } f \in D\left(B_{\alpha}\right), \\
D\left(B_{\alpha}\right) & =\left\{f \in \mathcal{L}_{\psi}: \alpha \circ f \in \mathcal{H}_{\Theta}\right\} .
\end{aligned}
$$

We assume that $\alpha=\bar{\alpha}$ and we set

$$
A_{\alpha}=B_{\alpha}^{*}
$$

By analogy to the case of defect indices $(1,1)$ we also have the following.

Theorem 3.3. The operator $B_{\alpha}$ is densely defined and closed.

Theorem 3.4. The operator $A_{\alpha}$ admits defect indices $(m, m)$ if and only if

$$
\varphi_{\lambda}^{(k)}=(\alpha-\lambda) \circ \Theta_{k} \in \mathcal{L}_{\psi}, \quad k=1, \ldots, m .
$$

In this case the functions $\varphi_{\lambda}^{(k)}(k=1, \ldots, m)$ are linearly independent and generate the defect space $\mathcal{N}_{\bar{\lambda}}$. 


\section{REFERENCES}

[1] M. B. Abrahamse, Multiplication operators, Lecture Notes in Mathematics 693 (1993), 17-36.

[2] N. I. Akhiezer and I. M. Glazman, Theory of Linear Operators in Hilbert Space, Dover Books on Mathematics, Dover Publications, New York, 1993.

[3] S. M. Bahri, On the extension of a certain class of Carleman operators, Z. Anal. Anwend. 26 (2007), 57-64.

[4] S. M. Bahri, Spectral properties of a certain class of Carleman operators, Arch. Math. (Brno) 43 (2007), 163-175.

[5] S. M. Bahri, On convex hull of orthogonal scalar spectral functions of a Carleman operator, Bol. Soc. Parana. Mat. (3) 26(1-2) (2008), 9-18.

[6] S. M. Bahri, On the localization of the spectrum for quasi-selfadjoint extensions of a Carleman operator, Math. Bohem. 137 (2012), 249-258.

[7] M. K. Belbahri, Series de Laurent formelles, Office des Publications Universitaires, Alger, 1981.

[8] T. Carleman, Sur les Equations Intégrales Singulières à Noyau Réel et Symétrique, Uppsala Almquwist Wiksells Boktryckeri, Uppsala, 1923.

[9] V. B. Korotkov, Integral Operators with Carleman Kernels (in Russian), Nauka, Novosibirsk, 1983.

[10] G. I. Tagronski, On Carleman integral operators, Proc. Amer. Math. Soc. 18 (1967), 450-456.

[11] J. Weidman, Carleman operators, Manuscripts Math. 2 (1970), 1-38.

[12] J. Weidman, Linear Operators in Hilbert Spaces, Spring Verlag, New-York Heidelberg Berlin, 1980.

${ }^{1}$ Laboratory of Pure and Applied Mathematics,

AbDelhamid IBN Badis University,

BP 227, Mostaganem 27000, Algeria

E-mail address: sidimohamed.bahri@univ-mosta.dz 